Теорія Ймовір. та Матем. Статист. Вип. 79, 2008
Theor. Probability and Math. Statist.

No. 79, 2009, Pages 39-55

S 0094-9000(09)00779-0

Article electronically published on December 29, 2009

\title{
FRACTAL PROPERTIES OF SOME BERNOULLI CONVOLUTIONS
}

UDC 519.21

\author{
YA. V. GONCHARENKO, M. V. PRATSYOVYTYĬ, AND G. M. TORBIN
}

Abstract. We study the structure and topological, metric, and fractal properties of the distribution of the random variable

$$
\xi=\sum_{k=1}^{\infty} \xi_{k} a_{k}
$$

where $\sum_{k=1}^{\infty} a_{k}$ is a convergent series of positive terms $a_{k}$ such that

$$
a_{3 k-2}=a_{3 k-1}+a_{3 k},
$$

$a_{i} \geq a_{i+1}+a_{i+2}+\cdots, i \neq 3 k-2, k \in \mathbf{N}$, and where $\xi_{k}$ are independent random variables assuming two values 0 and 1 with the probabilities $p_{0 k}$ and $p_{1 k}$, respectively. We prove that the distribution of $\xi$ is either purely discrete or purely singularly continuous. We obtain the criteria for a distribution to belong to each of these types. The topological-metric structure of the distribution is studied in the continuous case. The main result of the paper describes the fractal properties of the distribution of the random variable $\xi$. The relations are obtained for the Hausdorff-Besicovitch spectrum (the minimal closed support) of the random variable $\xi$ and for the Hausdorff dimension of the corresponding probability measure $\mu_{\xi}$.

\section{INTRODUCTION}

Let $\mu_{\xi}$ be the probability measure generated by the random variable

$$
\xi=\sum_{k=1}^{\infty} \xi_{k} a_{k}
$$

where

$$
\sum_{k=1}^{\infty} a_{k}=a_{1}+a_{2}+\cdots+a_{k}+\cdots
$$

is a convergent series of positive terms, and $\xi_{k}$ is a sequence of independent random variables with the following distributions:

$$
\mathrm{P}\left\{\xi_{k}=0\right\}=p_{0 k} \geq 0 \quad \text { and } \quad \mathrm{P}\left\{\xi_{k}=1\right\}=p_{1 k} \geq 0, \quad p_{0 k}+p_{1 k}=1 .
$$

The properties of the measure $\mu_{\xi}$ are determined by those of an infinite stochastic matrix $\left\|p_{i k}\right\|$ and by the sequence $\left\{a_{k}\right\}$ and do not depend on the sum $r_{0}$ of the series (2)

2000 Mathematics Subject Classification. Primary 60G30, 11K55, 28A80.

Key words and phrases. Bernoulli convolution, singularly continuous probability distribution, Hausdorff-Besicovitch dimension, Hausdorff dimension of a distribution (measure), the set of incomplete sums of a series.

The first author is supported by the grant DFG 436 113/80.

The second author is supported by the grants DFG 436 UKR 113/78 and DFG 436 113/80.

The third author is supported by the grants DFG 436 UKR 113/78 and DFG $436113 / 80$ and by the Alexander von Humboldt Foundation. 
(if we agree that equivalent distributions have the same properties). The Jessen-Wintner theorem [4, 23. implies that $\xi$ has a pure distribution (either purely discrete, or purely absolutely continuous (with respect to the Lebesgue measure), or purely singularly continuous). The Lévy theorem [27] provides necessary and sufficient conditions for the discreteness; namely, a measure $\mu_{\xi}$ is discrete if and only if

$$
M=\prod_{k=1}^{\infty} \max \left\{p_{0 k}, p_{1 k}\right\}>0 .
$$

A criterion for the absolute continuity (as well as for the singularity) of the distribution of $\xi$ is not yet known.

Note that the distribution of the random variable $\xi$ is a generalization of the symmetric Bernoulli convolution, which is the distribution of the random variable $\zeta=\sum_{k=1}^{\infty} \zeta_{k} a_{k}$ where the random variables $\zeta_{k}$ assume values -1 and 1 with equal probabilities $\frac{1}{2}$. The symmetric Bernoulli convolution has been intensively studied since the 1930s [4, 13, 19, 21, 28, 30, 31, 36. Unfortunately an exhaustive study of these distributions has not been done so far even in the case of $a_{k}=\lambda^{k}, \lambda>\frac{1}{2}, p_{0 k}=\frac{1}{2}$, although this was done for some particular values of the parameter $\lambda$ (see [19, 21]). The paper [29] contains a survey of the problems and results concerning these distributions. Some applications in harmonic analysis, the theory of dynamical systems, etc. are discussed in [16, 29. A survey of results for Bernoulli convolutions (not necessarily symmetric) can be found in [5, 15, 18.

Recall [3] that if $M$ is a finite or infinite subset of the set of positive integer numbers $\mathbf{N}$, then the number

$$
x=x(M)=\sum_{n \in M} a_{n}
$$

is called an incomplete sum of series (2). It is clear that

$$
x(M)=\sum_{n=1}^{\infty} \varepsilon_{n} a_{n}, \quad \text { where } \quad \varepsilon_{n}= \begin{cases}1 & \text { if } n \in M, \\ 0 & \text { if } n \notin M .\end{cases}
$$

The set of all incomplete sums of series (2) is denoted by $\Delta^{\prime}$, that is,

$$
\Delta^{\prime}:=\left\{x: x=\sum_{n \in M} a_{n}, M \in 2^{N}\right\} .
$$

In particular, all partial sums $S_{m}=\sum_{i=1}^{m} a_{i}$ and all tails $r_{m}=\sum_{i=m+1}^{\infty} a_{i}$ of series (2) (but not only them, of course) belong to the set $\Delta^{\prime}$, and $\Delta^{\prime} \subseteq\left[0, r_{0}\right]$, where $r_{0}$ denotes the sum of series (2).

The topological properties of the set $\Delta^{\prime}$ are studied in detail (see [13]). This is not the case as far as the metric and fractal properties are concerned. The study of the metric and fractal properties is a separate problem; it is successfully solved for some particular classes of series (see, for example, [3, 9]). The authors are unaware whether necessary and sufficient conditions appear in the literature in the general case for the set $\Delta^{\prime}$ to be of a zero measure (with respect to the Lebesgue measure) and of a formula for evaluating its Hausdorff-Besicovitch dimension.

The distribution of the random variable $\xi$ is a probability distribution defined on the set $\Delta^{\prime}$ of incomplete sums of series (2). Moreover the spectrum $S_{\xi}$ of the distribution of $\xi$ coincides with $\Delta^{\prime}$ if $p_{i k}>0$ for all $i \in\{0,1\}, k \in \mathbf{N}$.

The topological-metric and fractal properties of the Bernoulli convolution with $a_{k} \geq r_{k}$ for all $k \in \mathbf{N}$ are well studied [4, 5, 18, 29, 34, 35. A relative simplicity of this case is explained by the fact that, for an arbitrary point $u$ of the set $\Delta^{\prime}$, there are at most two subsets of the set of positive integer numbers such that $u=x\left(M_{i}\right)$. 
If the spectrum $S_{\xi}$ of the random variable $\xi$ contains at least one point $u$ such that the equality $u=x\left(M_{i}\right)$ holds for an infinite sequence of pairwise different sets $M_{i}$, then the distribution of $\xi$ is called a Bernoulli convolution with essential intersections. Such distributions are more complicated and hence less studied. Nowadays many scientists investigate these distributions (see [3, 29, 32]). The distributions considered below belong to the class of Bernoulli convolution with essential intersections.

In this paper, we study the type and topological-metric and fractal properties of the distribution of the random variable $\xi$ if series (2) is such that

$$
\left\{\begin{array}{l}
a_{3 k-2}=a_{3 k-1}+a_{3 k}, \\
r_{3 k-1} \leq a_{3 k-1}, \\
r_{3 k} \leq a_{3 k},
\end{array} \quad k \in \mathbf{N} .\right.
$$

In Section 2, we introduce the so-called cylindrical representation of numbers and that of the random variable $\xi$ defined by (11) and study the "geometry" of this representation. In Section 3, we discuss the case where $\xi$ is a random variable with independent $\widetilde{Q}_{-}$ symbols and where the matrix of distributions of its $\widetilde{Q}$-symbols can be explicitly written in terms of the elements of the initial matrix $\left\|p_{i k}\right\|$. In Section 4 , we use $\widetilde{Q}$-representation of the random variable $\xi$ and prove that the distribution of $\xi$ is purely singular in the continuous case. This completely solves the problem on the Lebesgue structure of the distribution of $\xi$.

Section 5 is devoted to studies of the structure of the random variable $\xi$ whose distribution is singular. We prove that the distribution of $\xi$ is purely singular and belongs to one of the three types, namely either to $S$-, or to $C$-, or to $K$-type, and give criteria for belonging to each of these types.

Section 6 is the main section in this paper. We study the fractal properties of the distribution of the random variable $\xi$. We solve the problem on the Hausdorff-Besicovitch dimension of the spectrum of the distribution of $\xi$ and the problem on the Hausdorff dimension of the distribution of $\xi$; namely, we answer the question on the "minimal" Hausdorff-Besicovitch dimension for which supports of the distribution of the random variable $\xi$ exist.

\section{The CYLINDRICAL REPRESENTATION OF THE RANDOM VARIABLE $\xi$}

In what follows we assume that the terms of series (2) satisfy conditions (3). The sum of series (2) is denoted by $r_{0}=1$. Thus the set $\Delta^{\prime}$ of its incomplete sums is well defined.

The set $\Delta_{c_{1} \ldots c_{m}}^{\prime}$ that contains incomplete sums of series (2) of the form

$$
\sum_{n=1}^{m} c_{n} a_{n}+\sum_{n=m+1}^{\infty} \varepsilon_{n} a_{n}, \quad \text { where } \varepsilon_{n} \in\{0,1\}
$$

is called the cylinder of rank $m$ with the base $c_{1}, \ldots, c_{m}$.

The segment

$$
\Delta_{c_{1} \ldots c_{m}}=\left[\inf \Delta_{c_{1} \ldots c_{m}}^{\prime}, \sup \Delta_{c_{1} \ldots c_{m}}^{\prime}\right]=\left[\sum_{n=1}^{m} c_{n} a_{n}, r_{m}+\sum_{n=1}^{m} c_{n} a_{n}\right]
$$

is called the cylindrical segment of rank $m$ with the base $c_{1}, \ldots, c_{m}$. The interval with the same endpoints as in $\Delta_{c_{1} \ldots c_{m}}$ is denoted by $\nabla_{c_{1} \ldots c_{m}}$ and is called the cylindrical interval of rank $m$ with the base $c_{1}, \ldots, c_{m}$.

Depending on the sequence $\left\{a_{n}\right\}$ and the set $\left(c_{1}, \ldots, c_{m}\right)$ both cases are possible where $\Delta_{c_{1} \ldots c_{m}}^{\prime}$ and $\Delta_{c_{1} \ldots c_{m}}$ coincide or where they do not coincide. Nevertheless, $\Delta_{c_{1} \ldots c_{m}}^{\prime} \subset$ $\Delta_{c_{1} \ldots c_{m}}$ in either case. 
The above definitions imply the following properties of the cylindrical sets:

1) $\inf \Delta_{c_{1} \ldots c_{m}}=\inf \Delta_{c_{1} \ldots c_{m}}^{\prime}, \sup \Delta_{c_{1} \ldots c_{m}}=\sup \Delta_{c_{1} \ldots c_{m}}^{\prime}$.

2) $\Delta_{c_{1} \ldots c_{m}}=\Delta_{c_{1} \ldots c_{m} 0} \cup \Delta_{c_{1} \ldots c_{m} 1}, \Delta_{c_{1} \ldots c_{m}}^{\prime}=\Delta_{c_{1} \ldots c_{m} 0}^{\prime} \cup \Delta_{c_{1} \ldots c_{m} 1}^{\prime}$.

3) $\inf \Delta_{c_{1} \ldots c_{m}}=\inf \Delta_{c_{1} \ldots c_{m} 0}<\inf \Delta_{c_{1} \ldots c_{m} 1}, \sup \Delta_{c_{1} \ldots c_{m}}=\sup \Delta_{c_{1} \ldots c_{m} 1}$.

4) $\left|\Delta_{c_{1} \ldots c_{m}}\right|=r_{m} \rightarrow 0, m \rightarrow \infty$.

5) $\bigcap_{m=1}^{\infty} \Delta_{c_{1} \ldots c_{m}}=\bigcap_{m=1}^{\infty} \Delta_{c_{1} \ldots c_{m}}^{\prime} \equiv \Delta_{c_{1} \ldots c_{m} \ldots}=x \in \Delta^{\prime} \subset[0,1]$

It is easy to see that

6) $\frac{\left|\Delta_{c_{1} \ldots c_{m} c}\right|}{\left|\Delta_{c_{1} \ldots c_{m}}\right|}=\frac{r_{m+1}}{a_{m+1}+r_{m+1}}=\frac{1}{\delta_{m+1}+1}$, where $\delta_{m+1}=\frac{a_{m+1}}{r_{m+1}}$.

7) $\Delta_{c_{1} \ldots c_{m}}=\Delta_{s_{1} \ldots s_{k}} \Longleftrightarrow\left\{\begin{array}{l}m=k, \\ \sum_{n=1}^{m}\left(c_{n}-s_{n}\right) a_{n}=0 .\end{array}\right.$

8) $\Delta_{c_{1} \ldots c_{m} 0} \cap \Delta_{c_{1} \ldots c_{m} 1}= \begin{cases}\Delta_{c_{1} \ldots c_{m} 011}=\Delta_{c_{1} \ldots c_{m} 100} & \text { if } m=3 k \\ \Delta_{c_{1} \ldots c_{m} 10 \ldots 0 \ldots} & \text { if } a_{m+1}=r_{m+1}, \\ \varnothing & \text { if } a_{m+1}>r_{m+1}\end{cases}$

Corollary. $\left|\Delta_{c_{1} \ldots c_{m} 0} \cap \Delta_{c_{1} \ldots c_{m} 1}\right|= \begin{cases}r_{m+3} & \text { if } m \in\{3 k\}, \\ 0 & \text { if } m \notin\{3 k\} .\end{cases}$

It follows from the definitions of the cylindrical sets (cylinders and segments) and from properties 2,4 , and 5 that, for any arbitrary sequence $\left\{c_{k}\right\}, c_{k} \in\{0,1\}$,

$$
\Delta_{c_{1}} \supset \Delta_{c_{1} c_{2}} \supset \cdots \supset \Delta_{c_{1} \ldots c_{k}} \supset \cdots \quad \text { and } \quad \Delta_{c_{1}}^{\prime} \supset \Delta_{c_{1} c_{2}}^{\prime} \supset \cdots \supset \Delta_{c_{1} \ldots c_{k}}^{\prime} \supset \cdots .
$$

Moreover, there exists a unique number $x \in[0, r]$ such that

$$
x=\bigcap_{m=1}^{\infty} \Delta_{c_{1} \ldots c_{m}}=\bigcap_{m=1}^{\infty} \Delta_{c_{1} \ldots c_{m}}^{\prime}=\sum_{k=1}^{\infty} c_{k} a_{k} .
$$

Representation (4) of a number $x$ is called cylindrical. Equality (4) is formally written as $x=\Delta_{c_{1} \ldots c_{m} \ldots}$ and is called the cylindrical representation of a number (point) $x$. The set of all points $x \in[0,1]$ that have the cylindrical representation coincides with the set of incomplete sums of series (2).

The following assertion follows directly from the definition of the cylindrical representation of a number: the numbers $u=\Delta_{c_{1} \ldots c_{m} \ldots}$ and $v=\Delta_{s_{1} \ldots s_{m} \ldots}$ coincide if and only if

$$
\sum_{i=1}^{\infty}\left(c_{i}-s_{i}\right) a_{i}=0
$$

The following results support the conjecture that the distribution of the random variable $\xi$ belongs to the class of Bernoulli convolutions with essential intersections. Conditions (3) include an interesting case studied in the paper [3. It is proved in [3] that there exists only one convergent series with positive terms such that

$$
\left\{\begin{array}{l}
a_{3 k-2}=a_{3 k-1}+a_{3 k} \\
r_{3 k-1}=a_{3 k-1} \\
r_{3 k}=a_{3 k}
\end{array}\right.
$$

for all $k \in \mathbf{N}$. In this case, $a_{3 k-2}=\frac{3}{7^{k}}, a_{3 k-1}=\frac{2}{7^{k}}$, and $a_{3 k}=\frac{1}{7^{k}}, k \in \mathbf{N}$, and almost every point (in the sense of the Lebesgue measure) of the set of incomplete sums of 
the series has a continuum of different cylindrical representations. At the same time, there are points that have a unique representation or a finite or infinite set of different representations.

Remark. The random variable (11) can be represented in the following form:

$$
\xi=\Delta_{\xi_{1} \xi_{2} \ldots \xi_{k} \ldots}^{\prime}=\Delta_{\xi_{1} \xi_{2} \ldots \xi_{k} \ldots}
$$

called the cylindrical representation of the random variable $\xi$.

The type of the distribution of $\xi$ is sometimes determined by the properties of series (2). The following result is helpful in such a case.

Theorem 2.1 (3]). If series (2) satisfies conditions (3), then the Lebesgue measure of the set $\Delta^{\prime}$ of its incomplete sums is evaluated as follows:

$$
\lambda\left(\Delta^{\prime}\right)=\lim _{k \rightarrow \infty} 7^{k} r_{3 k}=1-\sum_{k=1}^{\infty} 7^{k-1}\left[2\left(a_{3 k-1}-r_{3 k-1}\right)+2^{2}\left(a_{3 k}-r_{3 k}\right)\right] .
$$

\section{3. $\widetilde{Q}$-REPRESENTATION OF THE RANDOM VARIABLE $\xi$}

Consider the $\widetilde{Q}$-representation of numbers of the interval $[0,1]$ related to the cylindrical representation of numbers determined by a series satisfying condition (31). We define the sequence $\left\{m_{k}\right\}$ by

$$
m_{k}=\left\{\begin{array}{l}
7 \quad \text { if } a_{3 k-1}=r_{3 k-1}, a_{3 k}=r_{3 k}, \\
9 \quad \text { if } a_{3 k-1}>r_{3 k-1}, a_{3 k}=r_{3 k}, \\
11 \quad \text { if } a_{3 k-1}=r_{3 k-1}, a_{3 k}>r_{3 k}, \\
13 \quad \text { if } a_{3 k-1}>r_{3 k-1}, a_{3 k}>r_{3 k},
\end{array} \quad k \in \mathbf{N} .\right.
$$

For all $k \in \mathbf{N}$, define the numbers

$$
\begin{gathered}
\delta_{k}=\frac{r_{3 k}}{r_{3 k-3}}, \quad \rho_{k}=\frac{a_{3 k-1}-r_{3 k-1}}{r_{3 k-3}}=\frac{r_{3 k-2}-2 r_{3 k-1}}{r_{3 k-3}}, \\
\sigma_{k}=\frac{a_{3 k}-r_{3 k}}{r_{3 k-3}}=\frac{r_{3 k-1}-2 r_{3 k}}{r_{3 k-3}}
\end{gathered}
$$

and the random column vector $\vec{q}_{k}=\left(q_{0 k}, q_{1 k}, \ldots, q_{\left(m_{k}-1\right) k}\right)$, where

1) for $m_{k}=7$,

$$
q_{i k}=\frac{1}{7}, \quad i \in\{0,1,2,3,4,5,6\}=B_{7},
$$

2) for $m_{k}=9$,

$$
q_{i k}= \begin{cases}\delta_{k} & \text { if } i \in\{0,1,3,4,5,7,8\}=B_{9}, \\ \rho_{k} & \text { if } i \in\{2,6\}\end{cases}
$$

3) for $m_{k}=11$,

$$
q_{i k}= \begin{cases}\delta_{k} & \text { if } i \in\{0,2,3,5,7,8,10\}=B_{11}, \\ \sigma_{k} & \text { if } i \in\{1,4,6,9\}\end{cases}
$$

4) for $m_{k}=13$,

$$
q_{i k}= \begin{cases}\delta_{k} & \text { if } i \in\{0,2,4,6,8,10,12\}=B_{13} \\ \rho_{k} & \text { if } i \in\{1,5,7,11\} \\ \sigma_{k} & \text { if } i \in\{3,9\}\end{cases}
$$


The "random matrix" $\widetilde{Q}=\left\|q_{i k}\right\|$ whose $k$-th column coincides with the random vector $\vec{q}_{k}$ determines the $\widetilde{Q}$-representation [4, 12] of numbers of the interval [0,1] defined as follows. Let $A_{k}=\left\{0,1, \ldots, m_{k}-1\right\}$. Consider a surjective mapping $f$ of the space of sequences $L=A_{1} \times A_{2} \times \cdots \times A_{k} \times \cdots$ to $[0,1]$ defined as follows:

$$
f\left(\left\{\gamma_{k}\right\}\right)=x=\beta_{\gamma_{1} 1}+\sum_{k=2}^{\infty}\left[\beta_{\gamma_{k} k} \prod_{i=1}^{k-1} q_{\gamma_{i} i}\right],
$$

where $\beta_{\gamma_{k} k}=\sum_{j=0}^{\gamma_{k}-1} q_{j k}$. The latter equality can be written more briefly as follows:

$$
x=\widetilde{\Delta}_{\gamma_{1} \ldots \gamma_{k} \ldots,}, \quad \gamma_{k} \in A_{k} .
$$

Expression (9) is called the $\widetilde{Q}$-representation of the number $x$, while $\gamma_{k}$ is called the $k$-th $\widetilde{Q}$-symbol of the number $x$. The segment

$$
\left[\widetilde{\Delta}_{c_{1} \ldots c_{n} 00 \ldots 0 \ldots,}, \widetilde{\Delta}_{c_{1} \ldots c_{n}\left(m_{n+1}-1\right)\left(m_{n+2}-1\right) \ldots\left(m_{n+k}-1\right) \ldots}\right]
$$

is called the $\widetilde{Q}$-cylindrical segment of rank $n$ with the base $c_{1}, \ldots, c_{n}$ and denoted by $\widetilde{\Delta}_{c_{1} \ldots c_{n}}$. It is clear that

$$
\begin{aligned}
& \text { 1) } \widetilde{\Delta}_{c_{1} \ldots c_{n}}=\bigcup_{c=0}^{m_{n+1}-1} \widetilde{\Delta}_{c_{1} \ldots c_{n} c}, \\
& \text { 2) } \sup \widetilde{\Delta}_{c_{1} \ldots c_{n} c}=\inf \widetilde{\Delta}_{c_{1} \ldots c_{n}(c+1)}, c \in\left\{0,1, \ldots, m_{n+1}-2\right\}, \\
& \text { 3) }\left|\widetilde{\Delta}_{c_{1} \ldots c_{n}}\right|=\prod_{i=1}^{n} q_{c_{i} i} .
\end{aligned}
$$

Note that the set of incomplete sums of series (2) satisfying conditions (3) coincides with the set

$$
C\left[\widetilde{Q},\left\{B_{m_{n}}\right\}\right]=\left\{x: x=\widetilde{\Delta}_{\gamma_{1} \ldots \gamma_{n} \ldots,}, \gamma_{n} \in B_{m_{n}}\right\} .
$$

It is not hard to prove that, for an arbitrary family $c_{1} c_{2}, \ldots, c_{3 k-1} c_{3 k}, c_{i} \in\{0,1\}$, there exists a unique family $\gamma_{1}, \ldots, \gamma_{k}, \gamma_{i} \in B_{m_{i}}$ such that $\Delta_{c_{1} c_{2} \ldots c_{3 k-1} c_{3 k}}=\widetilde{\Delta}_{\gamma_{1} \ldots \gamma_{k}}$ and $\gamma_{i}$ is a function $\psi$ of the three symbols $c_{3 i-2}, c_{3 i-1}$, and $c_{3 i}$. The triples 011 and 100 define the same value $\gamma_{i}\left(\gamma_{i}=3\right.$ for $m_{i}=7 ; \gamma_{i}=4$ for $m_{i}=9 ; \gamma_{i}=5$ for $m_{i}=11$; and $\gamma_{i}=6$ for $\left.m_{i}=13\right)$.

Since the cylinders of rank $3 k$ are disjoint or coincide, there exists a bijection between the set of cylindrical segments $\Delta_{c_{1} \ldots c_{3 k}}$ of rank $3 k$ and the set of $\widetilde{Q}$-cylinders $\widetilde{\Delta}_{\gamma_{1} \ldots \gamma_{k}}$, $\gamma_{k} \in B_{m_{k}}$, generated by the mapping $\psi\left(c_{3 i-2} c_{3 i-1} c_{3 i}\right)=\gamma_{i}$.

Let

$$
C=\{000,001,010,011,100,101,110,111\}, \quad C^{*}=C \backslash\{011,100\} .
$$

Define the matrix $\widetilde{P}=\left\|\widetilde{p}_{i k}\right\|, 0 \leq i \leq m_{k}-1, k=1,2, \ldots$, by

(1) if $i \in A_{k} \backslash B_{m_{k}}$, then

$$
\widetilde{p}_{i k}=0 ;
$$

(2) if $\gamma_{k}=\psi\left(c_{3 k-2} c_{3 k-1} c_{3 k}\right)$ and $011 \neq c_{3 k-2} c_{3 k-1} c_{3 k} \neq 100$, then

$$
\widetilde{p}_{\gamma_{k} k}=p_{c_{3 k-2}(3 k-2)} p_{c_{3 k-1}(3 k-1)} p_{c_{3 k} 3 k} ;
$$

(3) if $\gamma_{k}=\psi(011)=\psi(100)$, then

$$
\widetilde{p}_{\gamma_{k} k}=p_{0(3 k-2)} p_{1(3 k-1)} p_{1(3 k)}+p_{1(3 k-2)} p_{0(3 k-1)} p_{0(3 k)} .
$$


Let $\tau_{k}$ be a sequence of independent random variables assuming values $0,1, \ldots, m_{k}-1$ of the set $A_{k}$ with the probabilities $\widetilde{p}_{0 k}, \widetilde{p}_{1 k}, \ldots, \widetilde{p}_{m_{k}-1, k}$, respectively. Consider the random variable

$$
\widetilde{\xi}=f\left(\eta_{1}, \eta_{2}, \ldots, \eta_{k}, \ldots\right)=\beta_{\eta_{1} 1}+\sum_{k=2}^{\infty}\left[\beta_{\eta_{k} k} \prod_{i=1}^{k-1} q_{\eta_{i} i}\right]=: \widetilde{\Delta}_{\tau_{1} \ldots \tau_{k} \ldots} .
$$

Lemma 3.1. The random variables $\xi$ and $\widetilde{\xi}$ are identically distributed.

Proof. It is necessary to prove that

$$
\mathrm{P}\left\{\xi \in \Delta_{c_{1} \ldots c_{3 k}}\right\}=\mathrm{P}\left\{\tilde{\xi} \in \widetilde{\Delta}_{\gamma_{1} \ldots \gamma_{k}}\right\}, \quad \text { where } \gamma_{i}=\psi\left(c_{3 i-2} c_{3 i-1} c_{3 i}\right) \in B_{m_{i}} .
$$

We follow the method of mathematical induction. Let $k=1$. By the properties of cylindrical segments and in view of the independence of $\xi_{1}, \xi_{2}$, and $\xi_{3}$ for $c_{1} c_{2} c_{3} \in C^{*}$ we obtain

$$
\mathrm{P}\left\{\xi \in \Delta_{c_{1} c_{2} c_{3}}\right\}=\mathrm{P}\left\{\xi_{1}=c_{1}, \xi_{2}=c_{2}, \xi_{3}=c_{3}\right\}=p_{c_{1} 1} p_{c_{2} 2} p_{c_{3} 3},
$$

while for $c_{1} c_{2} c_{3} \in\{011,100\}$, it follows that

$$
\Delta_{c_{1} c_{2} c_{3}}=\Delta_{011}=\Delta_{100}
$$

and

$$
\begin{aligned}
\mathrm{P}\left\{\xi \in \Delta_{c_{1} c_{2} c_{3}}\right\} & =\mathrm{P}\left\{\xi_{1}=0, \xi_{2}=1, \xi_{3}=1 \vee \xi_{1}=1, \xi_{2}=0, \xi_{3}=0\right\} \\
& =p_{01} p_{12} p_{13}+p_{11} p_{02} p_{03}
\end{aligned}
$$

The equality

is obvious in this case.

$$
\mathrm{P}\left\{\xi \in \Delta_{c_{1} c_{2} c_{3}}\right\}=\mathrm{P}\left\{\widetilde{\xi} \in \widetilde{\Delta}_{\psi\left(c_{1} c_{2} c_{3}\right)}\right\}
$$

Assume that equality (11) holds for $k=n$. For $k=n+1$, consider the following two cases:

1) $c_{3 n+1} c_{3 n+2} c_{3 n+3} \in C^{*}$,

2) $c_{3 n+1} c_{3 n+2} c_{3 n+3} \in\{011,100\}$.

For the first case,

$$
\begin{aligned}
\mathrm{P}\{\xi & \left.\in \Delta_{c_{1} \ldots c_{3 n} c_{3 n+1} c_{3 n+2} c_{3 n+3}}\right\} \\
& =\mathrm{P}\left\{\xi \in \Delta_{c_{1} \ldots c_{3 n}}, \xi_{3 n+1}=c_{3 n+1}, \xi_{3 n+2}=c_{3 n+2}, \xi_{3 n+3}=c_{3 n+3}\right\} \\
& =\mathrm{P}\left\{\xi \in \Delta_{c_{1} \ldots c_{3 n}}\right\} p_{c_{3 n+1}(3 n+1)} p_{c_{3 n+2}(3 n+2)} p_{c_{3 n+3}(3 n+3)} .
\end{aligned}
$$

According to the induction assumption and to the definition of $\widetilde{p}_{\gamma_{k} k}$, the latter expression is equal to

$\mathrm{P}\left\{\widetilde{\xi} \in \widetilde{\Delta}_{\gamma_{1} \ldots \gamma_{n}}\right\} \cdot \widetilde{p}_{\gamma_{n+1}(n+1)}=\mathrm{P}\left\{\widetilde{\xi} \in \widetilde{\Delta}_{\gamma_{1} \ldots \gamma_{n} \gamma_{n+1}}\right\}$, where $\gamma_{n+1}=\psi\left(c_{3 n+1} c_{3 n+2} c_{3 n+3}\right)$.

Analogously, in the second case,

$$
\begin{aligned}
\mathrm{P}\{\xi \in & \left.\Delta_{c_{1} \ldots c_{3 n+3}}\right\} \\
= & \mathrm{P}\left\{\xi \in \Delta_{c_{1} \ldots c_{3 n}}\right\} \\
& \times \mathrm{P}\left\{\xi_{3 n+1}=0, \xi_{3 n+2}=1, \xi_{3 n+3}=1 \vee \xi_{3 n+1}=1, \xi_{3 n+2}=0, \xi_{3 n+3}=0\right\} \\
= & \mathrm{P}\left\{\xi \in \Delta_{c_{1} \ldots c_{3 n}}\right\}\left(p_{0(3 n+1)} p_{1(3 n+2)} p_{1(3 n+3)}+p_{1(3 n+1)} p_{0(3 n+2)} p_{0(3 n+3)}\right) \\
= & \mathrm{P}\left\{\widetilde{\xi} \in \widetilde{\Delta}_{\gamma_{1} \ldots \gamma_{n}}\right\} \cdot \widetilde{p}_{\gamma_{n+1}(n+1)}=\mathrm{P}\left\{\widetilde{\xi} \in \widetilde{\Delta}_{\gamma_{1} \ldots \gamma_{n} \gamma_{n+1}}\right\},
\end{aligned}
$$

where $\gamma_{n+1}=\psi(011)=\psi(100)$. Thus equality (11) holds for all $k \in \mathbf{N}$. 


\section{The Singularity OF THE Distribution OF $\xi$}

Theorem 4.1. The random variable $\xi$ has either a purely discrete (if $M>0$ ) or a purely singularly continuous (if $M=0$ ) distribution.

Proof. According to the latter lemma and Lévy's theorem, it is sufficient to prove that the random variable $\widetilde{\xi}$ has a singular distribution in the case of $M=0$.

It is known (see [12]) that the random variable $\widetilde{\xi}$, as a random variable having independent $\widetilde{Q}$-symbols, has an absolutely continuous distribution if and only if

$$
\prod_{k=1}^{\infty}\left(\sum_{i=0}^{m_{k}-1} \sqrt{q_{i k} \widetilde{p}_{i k}}\right)>0 \text {. }
$$

It is easy to see that

$$
\sum_{i=0}^{m_{k}-1} \sqrt{q_{i k} \widetilde{p}_{i k}}=\sum_{i \in B_{m_{k}}} \sqrt{q_{i k} \widetilde{p}_{i k}}=\sum_{\substack{i \in B_{m_{k}} \\ \widetilde{p}_{i k} \neq 0}} \sqrt{q_{i k} \widetilde{p}_{i k}}=\sqrt{\delta_{k}} \sum_{i: \widetilde{p}_{i k} \neq 0} \sqrt{\widetilde{p}_{i k}} .
$$

Condition (3) implies the inequality $\delta_{k} \leq \frac{1}{7}$; moreover, the equality here holds if and only if $m_{k}=7$.

Consider the function $\varphi\left(x_{0}, x_{1}, \ldots, x_{6}\right)=\sqrt{x_{0}}+\sqrt{x_{1}}+\cdots+\sqrt{x_{6}}$ in part $G$ of the hyperplane $x_{0}+x_{1}+\cdots+x_{6}=1$ belonging to the cube $[0,1]^{7}$.

Since

$$
\overrightarrow{\left(\sqrt{x_{0}}, \sqrt{x_{1}}, \ldots, \sqrt{x_{6}}\right)} \cdot \overrightarrow{(1,1, \ldots, 1)} \leq\left|\overrightarrow{\left(\sqrt{x_{0}}, \sqrt{x_{1}}, \ldots, \sqrt{x_{6}}\right)}\right| \cdot|\overrightarrow{(1,1, \ldots, 1)}|=\sqrt{7}
$$

and the function $\varphi$ is continuous in $G$, it attains the maximal value $\sqrt{7}$ at

$$
x_{0}=x_{1}=\cdots=x_{6}=\frac{1}{7}
$$

moreover, $\varphi\left(x_{0}, x_{1}, \ldots, x_{6}\right)<\sqrt{7}$ for all other points of the set $G$.

The condition

$$
\sqrt{\delta_{k}} \sum_{i: \widetilde{p}_{i k} \neq 0} \sqrt{\widetilde{p}_{i k}} \rightarrow 1, \quad k \rightarrow \infty,
$$

is necessary for the convergence of the product (12). Since $\delta_{k} \leq \frac{1}{7}$ and

$$
\sum_{i: \widetilde{p}_{i k} \neq 0} \sqrt{\widetilde{p}_{i k}} \leq \sqrt{7}
$$

the condition $\sum_{i: \widetilde{p}_{i k} \neq 0} \sqrt{\widetilde{p}_{i k}} \rightarrow \sqrt{7}, k \rightarrow \infty$, is necessary for the absolute continuity of the distribution of the random variable $\widetilde{\xi}$. The function $\varphi\left(x_{0}, x_{1}, \ldots, x_{6}\right)$ is continuous in $G$ and the global maximum of $\varphi$ is unique in $G$. Hence the condition

$$
\sum_{i: \widetilde{p}_{i k} \neq 0} \sqrt{\widetilde{p}_{i k}} \rightarrow \sqrt{7}, \quad k \rightarrow \infty,
$$

is equivalent to $\widetilde{p}_{i k} \rightarrow \frac{1}{7}$ for all $i \in B_{13}$. Therefore, if $\widetilde{\xi}$ is absolutely continuous, then $\tilde{p}_{i_{k} k} \rightarrow \frac{1}{7}, k \rightarrow \infty$, for an arbitrary sequence $\left\{i_{k}\right\}, i_{k} \in B_{m_{k}}$. In particular,

$$
\left\{\begin{array}{l}
\widetilde{p}_{0 k}=\widetilde{p}_{\psi(000) k}=p_{0(3 k-2)} p_{0(3 k-1)} p_{0(3 k)} \rightarrow \frac{1}{7}, \quad k \rightarrow \infty . \\
\widetilde{p}_{\psi(001) k}=p_{0(3 k-2)} p_{0(3 k-1)} p_{1(3 k)} \rightarrow \frac{1}{7},
\end{array}\right.
$$

It follows from (13) that $\frac{p_{0(3 k)}}{p_{1(3 k)}} \rightarrow 1$ and this is equivalent to $p_{0(3 k)} \rightarrow \frac{1}{2}$ as $k \rightarrow \infty$. One can show analogously that $p_{0(3 k-1)} \rightarrow \frac{1}{2}$ and $p_{0(3 k-2)} \rightarrow \frac{1}{2}$ as $k \rightarrow \infty$.

Then $\widetilde{p}_{0 k}=p_{0(3 k-2)} p_{0(3 k-1)} p_{0(3 k)} \rightarrow \frac{1}{8} \neq \frac{1}{7}$ and this contradicts (13). 
Therefore the random variable $\xi$ cannot have an absolutely continuous distribution and the statement of the theorem follows, since the distribution of this random variable is pure.

\section{TOpologicAl-MetriC StRUCture of THE Distribution OF THE RANDOM VARIABLE $\xi$}

Recall 4] that a singularly continuous distribution is called a distribution of the pure $S$-type if its support coincides with a union of intervals and every one of these intervals belongs to the spectrum. A singularly continuous distribution is called a distribution of the $C$-type if the Lebesgue measure of its spectrum is zero. A singularly continuous distribution is called a distribution of the $K$-type if its spectrum is a nowhere dense set of a positive Lebesgue measure.

Theorem 5.1. The continuous random variable $\xi(M=0)$ is of

- a singular distribution of the $S$-type if and only if there exists a number $k_{0} \in \mathbf{N}$ such that

$$
a_{3 k-1}-r_{3 k-1}=a_{3 k}-r_{3 k}=0 \quad \text { for all } k \geq k_{0}
$$

and the matrix $\left\|p_{i k}\right\|$ contains a finite number of zeros;

- a singular distribution of the C-type if and only if

$$
\sum_{k=1}^{\infty}\left[\frac{\left(a_{3 k-1}-r_{3 k-1}\right)}{r_{3 k-3}}+\frac{\left(a_{3 k}-r_{3 k}\right)}{r_{3 k-3}}+\varepsilon_{k} \frac{r_{3 k}}{r_{3 k-3}}\right]=+\infty,
$$

where $\varepsilon_{k}=1$ if there is at least one zero in the columns $3 k-2,3 k-1$, or $3 k$ of the matrix $\left\|p_{i k}\right\|$, and $\varepsilon_{k}=0$ otherwise;

- a singular distribution of the $K$-type if and only if series (15) converges and contains an infinite number of positive terms.

Proof. According to Theorem 4.1, the random variable $\xi$ has a singular distribution in the continuous case. Since $\xi$ is a random variable with independent $\widetilde{Q}$-symbols, we apply the result of [12] on the topological-metric structure of such random variables; namely, the distribution of $\xi$ is of the $S$-type if and only if the corresponding matrix $\widetilde{P}$ does not have an infinite number of columns containing zero elements. This is equivalent to the combination of condition (14) and the condition that the matrix $\left\|p_{i k}\right\|$ has a finite number of zeros.

The random variable $\xi$ has a distribution of the $C$-type if and only if

$$
\sum_{k=1}^{\infty} \sum_{i: \widetilde{p}_{i k}=0} q_{i k}=+\infty
$$

$($ see $[12])$.

Let

$$
t_{k}= \begin{cases}0 & \text { if the elements } p_{i_{1}(3 k-2)}, p_{i_{2}(3 k-1)}, p_{i_{3}(3 k)} \text { are nonzero, } \\ 3 & \text { if there is only one zero among } p_{i_{1}(3 k-2)}, p_{i_{2}(3 k-1)}, p_{i_{3}(3 k)}, \\ 5 & \text { if there are exactly two zeros among } p_{i_{1}(3 k-2)}, p_{i_{2}(3 k-1)}, p_{i_{3}(3 k)}, \\ 6 & \text { if there are three zeros among } p_{i_{1}(3 k-2)}, p_{i_{2}(3 k-1)}, p_{i_{3}(3 k)} .\end{cases}
$$

Since

$$
\sum_{i: \widetilde{p}_{i k}=0} q_{i k}=4 \frac{a_{3 k}-r_{3 k}}{r_{3 k-3}}+2 \frac{a_{3 k-1}-r_{3 k-1}}{r_{3 k-3}}+t_{k} \frac{r_{3 k}}{r_{3 k-3}},
$$

the random variable $\xi$ has a distribution of the $C$-type if and only if condition (15) holds. 
Finally, a random variable with independent symbols in the $\widetilde{Q}$-representation has a distribution of the $K$-type if and only if the matrix $\widetilde{P}$ contains an infinite number of columns with zero elements and

$$
\sum_{k=1}^{\infty} \sum_{i: \widetilde{p}_{i k}=0} q_{i k}<+\infty
$$

(see [12]). The latter two conditions are equivalent to the combination of the following two conditions:

$$
\left(a_{3 k}-r_{3 k}\right)+\left(a_{3 k-1}-r_{3 k-1}\right)+t_{k} \frac{r_{3 k}}{r_{3 k-3}}>0
$$

for infinitely many indices $k$ and

$$
\sum_{k=1}^{\infty} \frac{\left(a_{3 k-1}-r_{3 k-1}\right)+\left(a_{3 k}-r_{3 k}\right)+t_{k} r_{3 k}}{r_{3 k-3}}<+\infty .
$$

\section{Fractal properties of the distribution of the RANDOM VARiable $\xi$}

If the set of incomplete sums of series (2) is of zero Lebesgue measure, then the fractal measures and dimensions (Hausdorff measure, Hausdorff-Besicovitch dimension, and their generalizations) are more subtle characteristics of the capacity of the spectrum of the distribution of the random variable $\xi$. We recall the definitions of these important notions.

Let $E_{0}$ be some fixed subset of a metric space, $\Phi\left(E_{0}\right)$ be a family of subsets of the same metric space such that, for an arbitrary subset $E \subset E_{0}$ and for an arbitrary $\varepsilon>0$, there exists an at most countable $\varepsilon$-covering $\left\{E_{j}\right\}$ of the set $E$ with the properties that $E_{j} \in \Phi\left(E_{0}\right)$ and $\left|E_{j}\right| \leq \varepsilon$, where $|E|$ denotes the diameter of the set $E$. For all subsets $E \subset E_{0}$ and for all $\alpha>0$ and $\varepsilon>0$, we define the number

$$
m_{\varepsilon}^{\alpha}\left(E, \Phi\left(E_{0}\right)\right)=\inf _{\left|E_{j}\right| \leq \varepsilon}\left\{\sum_{j}\left|E_{j}\right|^{\alpha}\right\}
$$

where the infimum is evaluated over all at most countable $\varepsilon$-coverings of the set $E$ such that $E_{j} \in \Phi\left(E_{0}\right)$.

The number

$$
H^{\alpha}\left(E, \Phi\left(E_{0}\right)\right)=\lim _{\varepsilon \rightarrow 0} m_{\varepsilon}^{\alpha}\left(E, \Phi\left(E_{0}\right)\right)=\sup _{\varepsilon>0} m_{\varepsilon}^{\alpha}\left(E, \Phi\left(E_{0}\right)\right)
$$

is called the $\alpha$-dimensional Hausdorff measure of the set $E$ with respect to a given family of coverings $\Phi\left(E_{0}\right)$.

If $E_{0}$ is the unit interval and $\Phi\left(E_{0}\right)$ is the family of all subsets of [0,1], or the family of all open (closed) subsets of $[0,1]$, or the family of all subintervals of $[0,1]$, then we obtain the same measure, called Hausdorff measure. We denote it by $H^{\alpha}(E)$.

Definition 6.1. The positive number

$$
\alpha_{0}\left(E, \Phi\left(E_{0}\right)\right)=\sup \left\{\alpha: H^{\alpha}\left(E, \Phi\left(E_{0}\right)\right)=+\infty\right\}=\inf \left\{\alpha: H^{\alpha}\left(E, \Phi\left(E_{0}\right)\right)=0\right\}
$$

is called the Hausdorff-Besicovitch dimension of the set $E$ with respect to a given family of coverings $\Phi\left(E_{0}\right)$.

If $\Phi\left(E_{0}\right)$ is the family of all subsets of the interval $[0,1]$ or the family of all subintervals of $[0,1]$, then we simply write $\alpha_{0}(E)$. It is known [17 that, when evaluating the Hausdorff-Besicovitch dimension of subsets of the unit interval, one can restrict consideration to the case of coverings that include only $s$-adic intervals. 
The following auxiliary result shows that one can restrict consideration to a sufficiently narrower class of coverings when evaluating the Hausdorff-Besicovitch dimension of subsets of the set of incomplete sums of a series.

Let $\mathcal{A}_{n}$ be the family of cylindrical segments of rank $3 n$, that is,

$$
\mathcal{A}_{n}=\left\{E: E=\Delta_{\alpha_{1} \ldots \alpha_{3 n}}, \alpha_{i} \in\{0,1\}, i=1,2, \ldots, 3 n\right\}, \quad \mathcal{A}=\bigcup_{n=1}^{\infty} \mathcal{A}_{n} .
$$

Lemma 6.1. Let $E$ be an arbitrary subset of the set $\Delta^{\prime}$ of incomplete sums. When evaluating the Hausdorff-Besicovitch dimension of the set $E$, one can restrict consideration to the case of coverings of the family $\mathcal{A}$, that is, $\alpha_{0}(E, \mathcal{A})=\alpha_{0}(E)$.

Proof. The inequality $\alpha_{0}(E, \mathcal{A}) \geq \alpha_{0}(E)$ is obvious, since the evaluation of the premeasure $m_{\varepsilon}^{\alpha}(E, \mathcal{A})$ is reduced, generally speaking, to the evaluation of the infimum with respect to a narrower class of coverings as compared to the evaluation of the premeasure $m_{\varepsilon}^{\alpha}(E)$.

We prove the converse inequality. Let $\left\{E_{i}\right\}$ be an arbitrary $\varepsilon$-covering of the set $E \subset \Delta^{\prime}$ by intervals $E_{i}=\left(a_{i}, b_{i}\right), E_{i} \cap E \neq \varnothing$. For every $E_{i}$, there exists a cylindrical segment $\Delta_{n_{i}} \equiv \Delta_{\alpha_{1} \ldots \alpha_{3 n_{i}}} \in \mathcal{A}_{n_{i}}$ such that

1) $\Delta_{\alpha_{1} \ldots \alpha_{3 n_{i}}} \subset E_{i}$;

2) every one of the segments $\mathcal{A}_{n_{i}-1}$ does not belong to $E_{i}$.

The set $E_{i} \cap E$ belongs to at most 13 cylindrical segments of rank $3 n_{i}$ that are adjacent with $\Delta_{n_{i}}$. Thus $\left|\Delta_{n_{i}}\right|<\varepsilon$ and $13\left|\Delta_{n_{i}}\right|^{\alpha}<13\left|E_{i}\right|^{\alpha}$.

Hence $m_{\varepsilon}^{\alpha}(E, \mathcal{A}) \leq \sum_{i} 13\left|\Delta_{n_{i}}\right|^{\alpha} \leq \sum_{i} 13\left|E_{i}\right|^{\alpha}$ for all $\varepsilon>0$ and $\alpha>0$ and for an arbitrary $\varepsilon$-covering of the set $E$ by intervals $E_{i}$. Thus

$$
m_{\varepsilon}^{\alpha}(E, \mathcal{A}) \leq 13 \cdot m_{\varepsilon}^{\alpha}(E) \quad \text { and } \quad H^{\alpha}(E, \mathcal{A}) \leq 13 \cdot H^{\alpha}(E)
$$

whence the inequality $\alpha_{0}(E, \mathcal{A}) \leq \alpha_{0}(E)$ follows.

The spectrum of a probability distribution describes its properties rather roughly; however, the spectrum is an adequate characteristic for a narrower class of distributions. For example, the classical Cantor set is the spectrum of a discretely distributed Bernoulli convolution (corresponding to the case of $a_{k}=\frac{2}{3^{k}}$ and $p_{0 k}=\frac{1}{2^{k}}$ ) and, at the same time, the spectrum for every member of the continual family of pairwise orthogonal (and orthogonal to the Lebesgue measure, as well) Bernoulli convolutions $\xi_{p}$ generated by the series with $a_{k}=\frac{2}{3^{k}}$ and $\left.p_{0 k}=p \in(0,1)\right)$.

Much more information about the properties of a singular distribution can be obtained by analyzing fractal properties of all its Borel supports.

The number

$$
\operatorname{dim}_{H}(\tau)=\inf _{E \in B_{\tau}}\left\{\alpha_{0}(E), E \in \mathcal{B}\right\}
$$

is called the Hausdorff dimension of the distribution of a random variable $\tau$ where $\mathcal{B}$ is the Borel $\sigma$-algebra of subsets of the real axis and where $B_{\tau}$ is the class of all possible supports (not necessarily closed) of the random variable $\tau$; that is,

$$
B_{\tau}=\left\{E: E \in \mathcal{B}, P_{\tau}(E)=1\right\}
$$

If $\tau$ is a discrete random variable, then obviously $\operatorname{dim}_{H}(\tau)=0$. Further, if $\tau$ is an absolutely continuous random variable, then $\operatorname{dim}_{H}(\tau)=1$. Finally, we have

$$
0 \leq \operatorname{dim}_{H}(\tau) \leq 1
$$

in the case of a singular random variable $\tau$, and moreover the following general bound holds:

$$
\operatorname{dim}_{H}(\nu) \leq \alpha_{0}\left(S_{\nu}\right)
$$


Now we evaluate the Hausdorff dimension of the distribution of the random variable (11). First we recall the following definition.

Let $\nu$ be a continuous probability measure on Borel subsets of the unit interval $[0,1]$, $E_{0}$ be a fixed subset of the unit interval, and let $\Phi\left(E_{0}\right)$ be a family of subintervals of $[0,1]$ such that, given a subset $E \subset E_{0}$ and an arbitrary $\varepsilon>0$, there exists an at most countable $(\nu-\varepsilon)$-covering $\left\{E_{j}\right\}$ of the set $E$ such that $E_{j} \in \Phi\left(E_{0}\right)$ and $\nu\left(E_{j}\right) \leq \varepsilon$. Then the $(\nu-\alpha)$-Hausdorff measure of the subset $E$ is defined as follows:

$$
H^{\alpha}\left(E, \nu, \Phi\left(E_{0}\right)\right)=\lim _{\varepsilon \rightarrow 0}\left\{\inf _{\nu\left(E_{j}\right) \leq \varepsilon} \sum_{j} \nu^{\alpha}\left(E_{j}\right)\right\}=\lim _{\varepsilon \rightarrow 0} H^{\alpha}(E, \nu, \varepsilon, \Phi),
$$

where $E_{j} \in \Phi\left(E_{0}\right)$ and $\bigcup_{j} E_{j} \supset E$.

Definition 6.2. The number $\alpha_{\nu}\left(E, \Phi\left(E_{0}\right)\right)=\inf \left\{\alpha: H^{\alpha}\left(E, \nu, \Phi\left(E_{0}\right)\right)=0\right\}$ is called the Hausdorff-Billingsley dimension of the set $E$ with respect to a measure $\nu$ and a family of coverings $\Phi\left(E_{0}\right)$.

If $E_{0}=[0,1]$ and $\nu$ is Lebesgue measure on $[0,1]$, then

$$
\alpha_{\nu}\left(E, \Phi\left(E_{0}\right)\right)=\alpha_{0}\left(E, \Phi\left(E_{0}\right)\right)
$$

Put

$$
h_{k}=-\sum_{\gamma_{k} \in B_{m_{k}}} \tilde{p}_{\gamma_{k} k} \ln \tilde{p}_{\gamma_{k} k}
$$

If $\widetilde{p}_{\gamma_{i} i}=0$, then we agree that $\widetilde{p}_{\gamma_{i} i} \ln \widetilde{p}_{\gamma_{i} i}=0$.

Theorem 6.1. The Hausdorff dimension of the distribution of the random variable $\xi$ is equal to

$$
\operatorname{dim}_{H}(\xi)=\varliminf_{k \rightarrow \infty} \frac{h_{1}+h_{2}+\cdots+h_{k}}{-\ln r_{3 k}}
$$

Proof. Recall that the distributions of the random variables $\xi$ and $\widetilde{\xi}$ coincide. One can restrict consideration to the case of supports that are subsets of the spectrum of $\xi$ when evaluating the Hausdorff dimension of its distribution.

Let $\Delta_{n}^{\widetilde{Q}}(x)=\Delta_{\alpha_{1}(x) \alpha_{2}(x) \ldots \alpha_{n}(x)}^{\widetilde{Q}}$ be a $\widetilde{Q}$-cylindrical segment of rank $n$ containing a point $x$ of the spectrum $S_{\xi}$ and let $\mu$ be the probability measure corresponding to the distribution of the random variable $\xi$; that is,

$$
\mu(E)=\mathrm{P}\{\xi \in E\}
$$

for all $E \in \mathcal{B}$, where $\lambda$ is Lebesgue measure on $[0,1]$. Then

$$
\begin{gathered}
\mu\left(\Delta_{n}^{\widetilde{Q}}(x)\right)=\widetilde{p}_{\alpha_{1}(x) 1} \cdot \widetilde{p}_{\alpha_{2}(x) 2} \cdots \widetilde{p}_{\alpha_{n}(x) n}, \\
\lambda\left(\Delta_{n}^{\widetilde{Q}}(x)\right)=q_{\alpha_{1}(x) 1} \cdot q_{\alpha_{2}(x) 2} \cdots q_{\alpha_{n}(x) n}=r_{3 n} .
\end{gathered}
$$

Consider the ratio

$$
\frac{\ln \mu\left(\Delta_{n}^{\widetilde{Q}}(x)\right)}{\ln \lambda\left(\Delta_{n}^{\widetilde{Q}}(x)\right)}=\frac{\sum_{j=1}^{n} \ln \widetilde{p}_{\alpha_{j}(x) j}}{\ln r_{3 n}}
$$

If $x=\Delta_{\alpha_{1}(x) \alpha_{2}(x) \ldots \alpha_{n}(x) \ldots}^{\widetilde{Q}}$ is chosen at random according to the probabilities

$$
\mathrm{P}\left(\alpha_{j}(x)=i\right)=\widetilde{p}_{i j}
$$

(that is, the distribution of the random variable $x$ is described by the measure $\mu$ ), then

$$
\left\{\eta_{j}\right\}=\left\{\eta_{j}(x)\right\}:=\left\{\ln \widetilde{p}_{\alpha_{j}(x) j}\right\}
$$


is a sequence of independent random variables with the following distributions:

$$
\begin{gathered}
\mathrm{P}\left\{\eta_{j}=\ln \widetilde{p}_{i j}\right\}=\widetilde{p}_{i j}, \quad i=0, \ldots, m_{j}-1, \\
\mathrm{E} \eta_{j}=\sum_{i=0}^{m_{j}-1} \widetilde{p}_{i j} \ln \widetilde{p}_{i j}=-h_{j}, \quad\left|h_{j}\right| \leq \ln 7, \\
\mathrm{E} \eta_{j}^{2}=\sum_{i=0}^{m_{j}-1} \widetilde{p}_{i j} \ln ^{2} \widetilde{p}_{i j} \leq c_{0}<\infty,
\end{gathered}
$$

where the constant $c_{0}$ does not depend on $j$, since the function $\varphi(x)=x \ln ^{2} x$ is bounded in the interval $[0,1]$. Thus the Kolmogorov strong law of large numbers implies that

$$
\lim _{n \rightarrow \infty} \frac{\left(\eta_{1}+\eta_{2}+\cdots+\eta_{n}\right)-\mathrm{E}\left(\eta_{1}+\eta_{2}+\cdots+\eta_{n}\right)}{n}=0
$$

for $\mu$-almost all points $x \in[0,1]$.

Note that $\mathrm{E}\left(\eta_{1}+\eta_{2}+\cdots+\eta_{n}\right)=\mathrm{E} \eta_{1}+\mathrm{E} \eta_{2}+\cdots+\mathrm{E} \eta_{n}=-\left(h_{1}+h_{2}+\cdots+h_{n}\right)$.

Put $H_{n}:=h_{1}+h_{2}+\cdots+h_{n}$ and $D=\varliminf_{n \rightarrow \infty} \frac{H_{n}}{-\ln r_{3 n}}$. Consider the set

$$
\begin{aligned}
T & =\left\{x: \lim _{n \rightarrow \infty}\left(\frac{\eta_{1}(x)+\eta_{2}(x)+\cdots+\eta_{n}(x)}{\ln r_{3 n}}-\frac{H_{n}}{-\ln r_{3 n}}\right)=0\right\} \\
& =\left\{x: \lim _{n \rightarrow \infty} \frac{\frac{\left(\eta_{1}(x)+\eta_{2}(x)+\cdots+\eta_{n}(x)\right)-M\left(\eta_{1}(x)+\eta_{2}(x)+\cdots+\eta_{n}(x)\right)}{n}}{\frac{1}{n} \ln r_{3 n}}=0\right\} .
\end{aligned}
$$

Since $r_{3 n} \leq \frac{1}{7^{n}}$, the product $\frac{1}{n} \ln r_{3 n}$ is separated from zero. Thus $\mu(T)=1$, whence $\alpha_{\mu}(T, \mathcal{A})=1$.

Consider the following sets:

$$
\begin{aligned}
T_{1} & =\left\{x: \varliminf_{n \rightarrow \infty}\left(\frac{\eta_{1}(x)+\eta_{2}(x)+\cdots+\eta_{n}(x)}{\ln r_{3 n}}-\frac{H_{n}}{-\ln r_{3 n}}\right)=0\right\}, \\
T_{2} & =\left\{x: \varliminf_{n \rightarrow \infty}\left(\frac{\eta_{1}(x)+\eta_{2}(x)+\cdots+\eta_{n}(x)}{\ln r_{3 n}}\right) \leq \frac{\lim }{n \rightarrow \infty} \frac{H_{n}}{-\ln r_{3 n}}\right\} \\
& =\left\{x: \varliminf_{n \rightarrow \infty} \frac{\ln \mu\left(\Delta_{n}^{\tilde{Q}}(x)\right)}{\ln \lambda\left(\Delta_{n}^{\widetilde{Q}}(x)\right)} \leq \underline{\lim } \frac{H_{n}}{-\ln r_{3 n}}\right\}, \\
T_{3} & =\left\{x: \varliminf_{n \rightarrow \infty} \frac{\eta_{1}(x)+\eta_{2}(x)+\cdots+\eta_{n}(x)}{\ln r_{3 n}} \geq \underline{\lim _{n \rightarrow \infty}} \frac{H_{n}}{-\ln r_{3 n}}\right\} \\
& =\left\{x: \varliminf_{n \rightarrow \infty} \frac{\ln \mu\left(\Delta_{n}^{\widetilde{Q}}(x)\right)}{\ln \lambda\left(\Delta_{n}^{\widetilde{Q}}(x)\right)} \geq \underline{\lim } \frac{H_{n}}{-\ln r_{3 n}}\right\} .
\end{aligned}
$$

It is obvious that $T \subset T_{1}$. One can prove that $T_{1} \subset T_{3}$ and $T \subset T_{2}$ (see, for example, [14]).

By Theorem 2.1 of [17], $\alpha_{\lambda}\left(T_{2}, \mathcal{A}\right) \leq D$. Taking into account the inclusion $T \subset T_{2}$, we obtain

$$
\alpha_{\lambda}(T, \mathcal{A}) \leq D
$$

Since

$$
T \subset T_{3}=\left\{x: \varliminf_{n \rightarrow \infty} \frac{\ln \mu\left(\Delta_{n}^{\widetilde{Q}}(x)\right)}{\ln \lambda\left(\Delta_{n}^{\widetilde{Q}}(x)\right)} \geq D\right\},
$$

Theorem 2.2 of [17] implies that

$$
\alpha_{\lambda}(T, \mathcal{A}) \geq D \cdot \alpha_{\mu}(T, \mathcal{A})=D \cdot 1=D .
$$


Thus $\alpha_{\lambda}(T, \mathcal{A})=D$. Since $\lambda$ is the Lebesgue measure on $[0,1]$,

$$
\alpha_{0}(T, \mathcal{A})=\alpha_{\lambda}(T, \mathcal{A})=D .
$$

We have $\alpha_{0}(T, \mathcal{A})=\alpha_{0}(T)$ by Lemma 6.1] Thus $\alpha_{0}(T)=D$.

Now we prove that the set $T$ defined above is the minimal-dimensional support of the measure $\mu$. Let $C$ be some support of the measure $\mu$, that is, $\mu(C)=1$. It is clear that $C_{1}=C \cap T$ is also a support of the measure $\mu$ and $C_{1} \subset C$. Thus $\alpha_{0}\left(C_{1}\right) \leq \alpha_{0}(C)$ and $C_{1} \subset T$. We prove that $\alpha_{0}\left(C_{1}\right)=\alpha_{0}(T)$.

Since $C_{1} \subset T$, we get $\alpha_{0}\left(C_{1}\right) \leq \alpha_{0}(T)=D$. On the other hand,

$$
C_{1} \subset T \subset T_{3}=\left\{x: \varliminf_{n \rightarrow \infty} \frac{\ln \mu\left(\Delta_{n}(x)\right)}{\ln \lambda\left(\Delta_{n}(x)\right)} \geq D\right\} .
$$

Thus Theorems 2.1 and 2.2 of [17] and Lemma 6.1] imply that

$$
\alpha_{0}\left(C_{1}\right)=\alpha_{\lambda}\left(C_{1}, \mathcal{A}\right) \geq D \cdot \alpha_{\mu}\left(C_{1}, \mathcal{A}\right)=D \cdot 1=D .
$$

Therefore $\alpha_{0}\left(C_{1}\right)=D=\alpha_{0}(T)$.

Corollary. If $\xi_{k}$ are independent identically distributed random variables, then the Hausdorff dimension of the distribution of the random variable $\xi$ is equal to

$$
\operatorname{dim}_{H}(\xi)=\lim _{k \rightarrow \infty} \frac{h_{1}}{-\frac{1}{k} \ln r_{3 k}}
$$

and, for an arbitrary series (2) satisfying condition (3), we have

$$
\operatorname{dim}_{H}(\xi) \leq \frac{11 \ln 2}{4 \ln 7} \approx 0.97956976
$$

Proof. If $\xi_{k}$ are independent identically distributed random variables, then relation (19) follows directly from relation (17).

Now we prove inequality (20). Recall that $r_{3 n} \leq \frac{1}{7^{n}}$ for an arbitrary series satisfying condition (3), whence $-\frac{1}{n} \ln r_{3 n} \geq \ln 7$.

On the interval $(0,1)$, consider the following function:

$$
\begin{aligned}
f(x)= & x^{3} \ln x^{3}+2 x^{2}(1-x) \ln \left(x^{2}(1-x)\right)+x(1-x) \ln (x(1-x)) \\
& +2 x(1-x)^{2} \ln \left(x(1-x)^{2}\right)+(1-x)^{3} \ln \left((1-x)^{3}\right) .
\end{aligned}
$$

One can prove that $f(x)$ has a continuous derivative and

$$
\lim _{x \rightarrow 0+} f(x)=\lim _{x \rightarrow 1-} f(x)=0 .
$$

This function attains its maximal value $\frac{11}{4} \ln 2$ at the point $x=\frac{1}{2}$.

Let $p_{0 k}=q \in(0,1)$ and $p_{1 k}=p=1-q$. It is not complicated to check that $h_{k}=f(q)$ in this case, whence $h_{k}=h_{1} \leq \frac{11}{4} \ln 2$. Thus

$$
\operatorname{dim}_{H}(\xi)=\varliminf_{k \rightarrow \infty}\left(h_{1} \cdot \frac{1}{-\frac{1}{k} \ln r_{3 k}}\right) \leq \frac{11}{4} \ln 2 \cdot \frac{1}{\ln 7} .
$$

Now we study the fractal properties of the spectrum of the random variable $\xi$. Let

$$
\hat{h}_{k}= \begin{cases}\ln 7 & \text { if there is no zero among } p_{i_{1}(3 k-2)}, p_{i_{2}(3 k-1)}, p_{i_{3}(3 k)}, \\ \ln 4 & \text { if there is exactly one zero among } p_{i_{1}(3 k-2)}, p_{i_{2}(3 k-1)}, p_{i_{3}(3 k)}, \\ \ln 2 & \text { if there are exactly two zeros among } p_{i_{1}(3 k-2)}, p_{i_{2}(3 k-1)}, p_{i_{3}(3 k)}, \\ 0 & \text { if there are exactly three zeros among } p_{i_{1}(3 k-2)}, p_{i_{2}(3 k-1)}, p_{i_{3}(3 k)} .\end{cases}
$$


Theorem 6.2. The Hausdorff-Besicovitch dimension of the spectrum of the distribution of the random variable $\xi$ is given by

$$
\alpha_{0}\left(S_{\xi}\right)=\varliminf_{k \rightarrow \infty} \frac{\hat{h}_{1}+\hat{h}_{2}+\cdots+\hat{h}_{k}}{-\ln r_{3 k}} .
$$

Proof. Consider the random variable $\hat{\xi}$ with independent $\widetilde{Q}$-symbols defined in the following way:

$$
\left\|\hat{q}_{i k}\right\|=\left\|q_{i k}\right\|
$$

$\hat{p}_{i k}=0$ if $p_{i k}=0$, and $\hat{p}_{i k}=\frac{1}{l_{k}}$ if $p_{i k}>0$, where $l_{k}$ is the number of nonzero elements in the column $k$ of the matrix $\widetilde{P}$. It is not hard to check that

(1) if there is no zero among $p_{i_{1}(3 k-2)}, p_{i_{2}(3 k-1)}, p_{i_{3}(3 k)}$, then $l_{k}=7$ and

$$
-\sum_{i} \hat{p}_{i k} \ln \left(\hat{p}_{i k}\right)=\hat{h}_{k}=\ln 7
$$

(2) if there is exactly one zero among $p_{i_{1}(3 k-2)}, p_{i_{2}(3 k-1)}, p_{i_{3}(3 k)}$, then $l_{k}=4$ and

$$
-\sum_{i} \hat{p}_{i k} \ln \left(\hat{p}_{i k}\right)=\hat{h}_{k}=\ln 4,
$$

(3) if there are exactly two zeros among $p_{i_{1}(3 k-2)}, p_{i_{2}(3 k-1)}, p_{i_{3}(3 k)}$, then $l_{k}=2$ and

$$
-\sum_{i} \hat{p}_{i k} \ln \left(\hat{p}_{i k}\right)=\hat{h}_{k}=\ln 2 \text {, }
$$

(4) if there are exactly three zeros among $p_{i_{1}(3 k-2)}, p_{i_{2}(3 k-1)}, p_{i_{3}(3 k)}$, then $l_{k}=1$ and

$$
-\sum_{i} \hat{p}_{i k} \ln \left(\hat{p}_{i k}\right)=\hat{h}_{k}=0 .
$$

It is obvious that $S_{\hat{\xi}}=S_{\xi}$, since $\left\|\hat{q}_{i k}\right\|=\left\|q_{i k}\right\|$ and $\hat{p}_{i j}=0$ if and only if $p_{i j}=0$. The random variable $\hat{\xi}$ is uniformly distributed in the spectrum $S_{\xi}$ of the random variable $\xi$. Hence $\alpha_{0}\left(S_{\xi}\right)=\alpha_{0}\left(S_{\hat{\xi}}\right)=\operatorname{dim}_{H}(\hat{\xi})$. The random variable $\hat{\xi}$, generally speaking, is not a random variable of the form (1), but it has independent $\widetilde{Q}$-symbols and moreover the corresponding matrix $\widetilde{Q}$ is the same for $\xi$ and $\hat{\xi}$.

Thus relation (17) remains true for the evaluation of the Hausdorff dimension of the distribution of the random variable $\hat{\xi}$, too. Applying this relation we obtain

$$
\alpha_{0}\left(S_{\xi}\right)=\operatorname{dim}_{H}(\hat{\xi})=\varliminf_{k \rightarrow \infty} \frac{\hat{h}_{1}+\hat{h}_{2}+\cdots+\hat{h}_{k}}{-\ln r_{3 k}} .
$$

Corollary 1. Let $\xi_{k}$ be independent random variables and $0<p_{0 k}<1$ for all $k \in \mathbf{N}$. Then the Hausdorff-Besicovitch dimension of the spectrum of the distribution of the random variable $\xi$ is given by

$$
\alpha_{0}\left(S_{\xi}\right)=\varliminf_{k \rightarrow \infty} \frac{\ln 7}{-\frac{1}{k} \ln r_{3 k}}=\varliminf_{k \rightarrow \infty} \log _{r_{3 k}} 7^{-k} .
$$

Corollary 2. The Hausdorff-Besicovitch dimension of the set $\Delta^{\prime}$ of incomplete sums of series (2) satisfying conditions (3) is given by

$$
\alpha_{0}\left(\Delta^{\prime}\right)=\varliminf_{k \rightarrow \infty} \log _{r_{3 k}} 7^{-k}
$$

Proof. Let $\xi_{k}$ be a sequence of independent identically distributed random variables assuming the values 0 and 1 with equal probabilities $p_{0 k}=p_{1 k}=\frac{1}{2}$. Then the spectrum of the random variable $\xi=\sum_{k=1}^{\infty} \xi_{k} a_{k}$ coincides with the set $\Delta^{\prime}$ of incomplete sums of series (2). Thus Corollary 2 follows from Corollary 1 . 


\section{BIBLIOGRAPHY}

1. Ya. V. Goncharenko, Convolutions of distributions of sums of random series of a special form, Naukov. Zapys. Nats. Dragomanov Pedagog. Univ. Fiz. Mat. Nauky (2003), no. 4, 216-232. (Ukrainian)

2. Ya. V. Goncharenko, M. V. Pratsyovytǐ̌, and G. M. Torbin, Topological-metric and fractal properties of a convolution of two singular distributions of random variables with independent binary digits, Teor. Imovir. Mat. Stat. 67 (2002), 9-19; English transl. in Theory Probab. Math. Statist. 67 (2003), 11-22. MR 1956615 (2003k:60032)

3. Ya. V. Goncharenko, M. V. Pratsyovytiŭ, G. M. Torbin, Topological-metric and fractal properties of the set of incomplete sums of a series of positive terms and distributions on it,Naukov. Chasopys Nats. Dragomanov Pedagog. Univ. Ser. 1, Fiz. Mat. Nauky (2005), no. 6, 210-224. (Ukrainian)

4. M. V. Pratsyovytiı̌, Fractal Approach in the Studies of Singular Distributions, National Dragomanov Pedagogical University, Kyiv, 1998. (Ukrainian)

5. M. V. Pratsyovytiĭ and G. M. Torbin, On a class of random variables of the Jessen-Wintner type, Dopov. Nats. Akad. Nauk Ukr. Mat. Prirodozn. Tekh. Nauki (1998), no. 4, 48-54. (Ukrainian) MR1699106 (2000g:60020)

6. G. M. Torbin, Fractal properties of distributions of random variables with independent $Q^{*}$ symbols, Naukov. Zapys. Nats. Dragomanov Pedagog. Univ. Fiz. Mat. Nauky (2002), no. 3, 363-375. (Ukrainian)

7. G. M. Torbin, Multifractal analysis of singularly continuous probability measures, Ukrain. Mat. Zh. 57 (2005), no. 5, 706-721; English transl. in Ukrainian Math. J. 57 (2005), no. 5, 837-857. MR2209816 (2007f:28010)

8. A. F. Turbin and M. V. Pratsyovytiŭ, Fractal Sets, Functions, Distributions, Naukova Dumka, Kiev, 1992. (Russian) MR1353239 (96f:28010)

9. T. Šalat, Hausdorff measure of linear sets, Czechoslovak Math. J. 11(86) (1961), 24-56. (Russian) MR 0153802 (27:3763)

10. S. Albeverio, Ya. Gontcharenko, M. Pratsiovytyı̆, and G. Torbin, Convolutions of distributions of random variables with independent binary digits, Random Oper. Stoch. Equ. 15 (2007), no. 1, 89-97. MR2316190(2008b:11089)

11. S. Albeverio, Ya. Gontcharenko, M. Pratsiovytyı̆, and G. Torbin, Jessen-Wintner type random variables and fractal properties of their distributions, Math. Nachr. 279 (2006), no. 15, 16191633. MR:2269312 (2008g:28034)

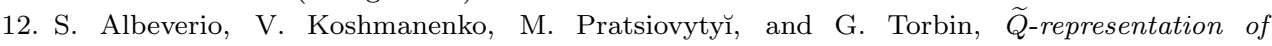
real numbers and fractal probability distributions, Preprint SFB-611, Bonn, http://front. math.ucdavis.edu/0308.5007 (submitted to J. Funct. Anal.).

13. S. Albeverio and G. Torbin, Image measures of infinite product measures and generalized Bernoulli convolutions, Naukov. Chasopys Nats. Dragomanov Pedagog. Univ. Ser. 1, Fiz. Mat. Nauky (2004), no. 5, 248-264.

14. S. Albeverio and G. Torbin, Fractal properties of singularly continuous probability distributions with independent $Q^{*}$-digits, Bull. Sci. Math. 129 (2005), no. 4, 356-367. MR2134126 (2006b:28013)

15. S. Albeverio and G. Torbin, On fine fractal properties of generalized infinite Bernoulli convolutions, Preprint SFB-611, vol. 340, 2007. Bull. Sci. Math. 132 (2008), 711-727. MR2474489

16. J. C. Alexander and D. Zagier, The entropy of a certain infinitely convolved Bernoulli measure, J. London Math. Soc. 44 (1991), 121-134. MR1122974 (92g:28035)

17. P. Billingsley, Hausdorff dimension in probability theory. II, Illinois J. Math. 5 (1961), 291-198. MR0120339 (22:11094)

18. M. Cooper, Dimension, measure and infinite Bernoulli convolutions, Math. Proc. Cambr. Phil. Soc. 124 (1998), 135-149. MR.1620520 (99h:28013)

19. P. Erdös, On a family of symmetric Bernoulli convolutions, Amer. J. Math. 61 (1939), 974-975. MR0000311(1:52a)

20. K. J. Falconer, Fractal Geometry, John Wiley \& Sons, 1990. MR 1102677 (92j:28008)

21. A. M. Garsia, Arithmetic properties of Bernoulli convolutions, Trans. Amer. Math. Soc. 102 (1962), 409-432. MR0137961 (25:1409)

22. R. Kershner and A. Wintner, On symmetric Bernoulli convolutions, Amer. J. Math. 57 (1935), 541-548. MR.1507093

23. B. Jessen and A. Wintner, Distribution functions and the Riemann zeta function, Trans. Amer. Math. Soc. 38 (1935), 48-88. MR1501802 
24. F. Ledrappier and A. Porzio, A dimension formula for Bernoulli convolutions, J. Statist. Phys. 76 (1994), 1307-1327. MR1298104 (95i:58111)

25. F. Ledrappier and A. Porzio, On the multifractal analysis of Bernoulli convolutions. I. Largedeviation results, J. Statist. Phys. 82 (1996), 367-395. MR.1372657 (97b:58088)

26. F. Ledrappier and A. Porzio, On the multifractal analysis of Bernoulli convolutions. II. Dimensions, J. Statist. Phys. 82 (1996), 397-420. MR1372658 (97b:58089)

27. P. Lévy, Sur les séries dont les termes sont des variables indépendantes, Studia Math. 3 (1931), 119-155.

28. R. Lyons, Seventy years of Rajchman measures, J. Fourier Anal. Appl., Kahane Special Issue (1995), 363-377. MR1364897 (97b:42019)

29. Y. Peres, W. Schlag, and B. Solomyak, Sixty years of Bernoulli convolutions in fractal geometry and stochastics II, Progress in Probab. 46 (2000), 39-65. MR 1785620 (2001m:42020)

30. Y. Peres and B. Solomyak, Absolute continuity of Bernoulli convolutions, a simple proof, Math. Res. Lett. 3 (1996), no. 2, 231-239. MR.1386842 (97f:28006)

31. Y. Peres and B. Solomyak, Self-similar measures and intersections of Cantor sets, Trans. Amer. Math. Soc. 350 (1998), no. 10, 4065-4087. MR1491873 (98m:26009)

32. Y. Peres, K. Simon, and B. Solomyak, Absolute continuity for random iterated function systems with overlaps, J. London Math. Soc. 74 (2006), no. 3, 739-756. MR2286443 (2007m:37053)

33. M. V. Pratsiovytyı and O. Y. Feshchenko, Topological-metrical and fractal properties of the distributions on the set of the incomplete sums of series of positive terms, Theory of Stochastic Processes 13 (29) (2007), no. 1-2, 205-224. MR2343824 (2009a:28025)

34. J. Reich, Some results on distributions arising from coin tossing, Ann. Probab. 10 (1982), no. 3, 780-786. MR659547 (83k:60026a)

35. J. Reich, When do weighted sums of independent random variables have a density-some results and examples, Ann. Probab. 10 (1982), no. 3, 787-798. MR659548(83k:60026b)

36. B. Solomyak, On the random series $\sum \pm \lambda^{n}$ (an Erdös problem), Ann. of Math. (2) 142 (1995), 611-625. MR:1356783 (97d:11125)

Department of Higher Mathematics, National Dragomanov Pedagogical University, Pirogova Street 9, Kyiv 01130, Ukraine

E-mail address: yan_a@ukr.net

Department of Higher Mathematics, National Dragomanov Pedagogical University, Pirogova Street 9, Kyiv 01130, Ukraine

E-mail address: prats4@yandex.ru

Department of Higher Mathematics, National Dragomanov Pedagogical University, PiroGova Street 9, Kyiv 01130, Ukraine

E-mail address: torbin@imath.kiev.ua

Received 27/NOV/2007

Translated by N. SEMENOV 